\title{
Redefiniendo la Educación Musical Inclusiva: Una revisión teórica
}

\author{
Santiago Díaz Santamaría ${ }^{1}$; Odet Moliner García
}

Recibido: 29 de abril de 2020 / Aceptado: 22 de mayo de 2020

Resumen. Más allá de la integración de las personas con necesidades especiales, la educación inclusiva parte de la consideración de la educación como un derecho de toda la ciudadanía. La educación musical comparte este derecho y se inspira en los principios de la inclusión. Con objeto de profundizar en los desafíos que presenta la educación musical desarrollada con un enfoque inclusivo, el objetivo de este estudio es presentar, a partir de la revisión de la literatura, la definición del concepto de Educación Musical Inclusiva (EMI). La revisión de la literatura científica a partir de la búsqueda en bases de datos especializadas, siguió el proceso de tres fases propuesto por Guirao, Olmedo y Ferrer (2008) finalizando con un análisis de contenido de tipo mixto. Como resultado se presenta una conceptualización de EMI que, sin obviar los aportes del enfoque terapéutico, incide en el enfoque didáctico centrándose en los factores de adaptabilidad y utilización de la música como recurso para lograr la inclusión educativa y social.

Palabras clave: educación musical; educación inclusiva; adaptabilidad; educación musical inclusiva

\section{[en] Redefining Inclusive Music Education: A Theoretical Review}

Abstract. Beyond the integration of people with special needs, inclusive education is a right of all citizens. Music education shares this right and is inspired by the principles of inclusion. In order to go deeper into the challenges presented by music education developed with an inclusive approach, the aim of this study is to present, based on a review of the literature, the definition of the concept of Inclusive Music Education (IME). The review of the scientific literature, based on the search in specialized databases, followed the three-phase process proposed by Guirao, Olmedo and Ferrer (2008) ending with a mixed content analysis. The result is a conceptualization of IME that, without ignoring the contributions of the therapeutic approach, emphasizes the didactic approach focusing on adaptability factors and the use of music as a resource to achieve educational and social inclusion.

Keywords: music education; inclusive education; adaptability; inclusive music education

Sumario. 1. Introducción. 2. Metodología. 3. Resultados 4. Conclusiones. 5. Referencias bibliográficas.

Cómo citar: Díaz-Santamaría, S. y Moliner, O. (2020). Redefiniendo la Educación Musical Inclusiva: Una revisión teórica. Revista Electrónica Complutense de Investigación en Educación Musical, 17, 21-31.

\section{Introducción}

La educación musical es susceptible de ser considerada como un derecho de todo el alumnado, considerando que es necesaria para asegurar la transmisión de un sustancial código de comunicación como es la música y también porque es un recurso eficaz para el desarrollo de las aptitudes individuales que inciden en la educación integral del ser humano (Monmany, 2004). Es por esta característica que la educación musical, a pesar de su carácter específico, no ha de ser ajena a la educación inclusiva la cual, definida y planteada también como un derecho, pretende la total integración de todo tipo de alumnado, incluso de aquel que posee necesidades especiales severas.

\footnotetext{
Conservatorio Profesional Joaquín Rodrigo de Sagunto

E-mail: al122347@alumail.uji.es

$2 \quad$ Universitat Jaume I

E-mail: molgar@uji.es
} 
Implementar la educación inclusiva en nuestros días es un imperativo docente que está avalado desde 2016 por el artículo 24 de la Convención de Derechos de las Personas con Discapacidad, siendo uno de los objetivos de la Agenda 2030 para el Desarrollo Sostenible. Concretamente, el objetivo 4 del plan de acción acordado por la ONU es garantizar una educación inclusiva, equitativa y de calidad y promover oportunidades de aprendizaje durante toda la vida para todos.

En el caso de la música, ya desde la antigüedad griega se ha considerado la práctica musical como un elemento que contribuye al desarrollo integral de la persona, pues favorece no solo el desarrollo de las capacidades artístico-creativas, sino también su integración sociocultural (Pérez y del Basto, 2011). La extensa proyección de las características del arte musical abarca aspectos determinantes de la inclusión social y educativa como son: la valoración positiva de la diversidad (Fernández y Epelde, 2014; Llopis, 2011; Pérez-Aldeguer, 2013), la resolución de conflictos (Cabedo-Mas, 2014a), el sentido de pertenencia (Bernabé-Villodre, 2012) y la creación de una cultura colaborativa (Howes, Davies, y Fox, 2009).

Asegurar la eficiencia de la educación inclusiva en todo ámbito académico incluye contemplar la docencia musical. Según estudios recientes (Wong y Chik, 2016), urge formar al profesorado especialista en los procedimientos y características de la educación inclusiva, pues, en ocasiones, la falta de formación condiciona el fracaso de la inclusión total. En esta relación dialéctica entre la educación inclusiva y la educación musical, el presente trabajo pretende indagar sobre la integración de ambos, analizando en qué medida podríamos hablar de una educación musical inclusiva como concepto a definir, o a redefinir, en su caso.

La revisión de estudios previos permite, por una parte, constatar la abundancia de trabajos que investigan el uso de la música como recurso de intervención terapéutica, acreditando su eficacia para la mejora de ciertos aspectos educativos (Bunt, 2003; Gurgenidze y Mamuladze, 2017; Pellitteri, 2000; Petruta-Maria, 2015; Rickson y McFerran, 2007) y, por otra, confirmar una escasez de aquellos que se centran en estudiar la educación musical desde la perspectiva de la educación inclusiva.

Como recurso de intervención terapéutica son varios los estudios que muestran cómo la musicoterapia beneficia a las personas con discapacidad o con alguna enfermedad, desde los cinco modelos principales de trabajo que recoge Sabbatella (2005, p. 136): "Creativa, Analítica, Conductista, Benenzon y Guided Imaginery and Music". Es el caso del estudio de revisión de Calleja-Bautista, Sanz-Cervera y Tárraga-Mínguez (2016) centrado en su efectividad en las personas con TEA, sobre todo para mejorar la comunicación e interacción social. También la revisión de Leggieri et al. (2019) evidencia los efectos positivos de la musicoterapia en personas con Alzheimer, sobre todo en cognición y conducta. Otros estudios se enmarcan en el ámbito educativo (Del Barrio, Sabbatella y Mercadal, 2019; Gfeller, 2000; Pérez y Sabbatella, 2014) y en el de la musicoterapia comunitaria en educación (McFerran y Rickson, 2014). Sin embargo, son menos frecuentes los estudios sobre musicoterapia que ponen el énfasis en los efectos en la inclusión social y educativa. Un ejemplo es el trabajo de Ouellet y Poliquin (2012) en el que la intervención con musicoterapia en un niño con TDAH procura una "experiencia armoniosa" que bien puede considerarse inclusiva por la relación de la misma con los lazos sociales que se dan en el ámbito escolar o por la idea comunitaria del grupo que conforma el contexto humano educativo.

Por otra parte, encontramos estudios que puede servir para dar respuesta a los nuevos retos que plantea el paradigma de la educación inclusiva, tanto en las aulas como en las sociedades desde un marco comunitario y de diversidad cultural (Rickson y McFerran, 2014). Esta línea nos conduce a los trabajos de Llopis (2011) y de Pérez-Aldeguer (2013) que, desde una concepción intercultural, recurren a los aspectos musicales como herramienta configuradora de una comunidad educativa en donde el sentido de pertenencia del individuo y su cohesión al grupo están directamente influenciados por el arte de los sonidos. Es comprensible que, a su vez, el recurso musical logre el equilibrio necesario entre el individuo y la comunidad, pues se puede considerar que, simultáneamente al fomento del sentido de pertenencia al grupo, la función social de la música afecta a la auto-identidad del individuo (North y Hargreaves, 2008) que no pierde sus rasgos propios en el proceso de inclusión en la comunidad. Esta comunidad, que se mantiene desde la convivencia pacífica, enfatiza la utilización de un código comunicativo universal como es la música, que se convierte en la piedra angular por su capacidad de transformar conflictos (Cabedo-Mas, 2014a) que muchas veces surgen en la sociedad y en el aula.

Otros estudios, como el de Burnard, Dillon, Rusinek, y Sæther, (2008), muestran las formas complejas en que los maestros logran la inclusión, referida a cómo todos los niños y niñas progresan y participan en las aulas de música superando desafíos derivados de su situación de pobreza, clase, raza, religión, patrimonio lingüístico y cultural o género.

Con objeto de indagar sobre las posibilidades de integración de la educación musical y la educación inclusiva, en nuestro trabajo indagatorio partimos de las aportaciones de Sabbatella (2008), autora que definió la educación musical inclusiva como un área emergente referida al

conjunto de estrategias y recursos utilizados para facilitar el acceso a la educación musical de todos los individuos atendiendo a sus características, intereses, capacidades y necesidades de aprendizaje (diferencias culturales, sociales, de género y personales) en contextos formales y no formales. (p. 260) 
Tomando como referencia tales aportaciones, el presente trabajo pretende, a partir de la revisión bibliográfica de la literatura, redefinir este tipo de educación, partiendo de la hipótesis de que vamos a encontrar estudios enmarcados en la didáctica de la educación musical con claras influencias terapéuticas.

\section{Metodología}

Se trata de una revisión sistemática de la literatura que tiene por objeto identificar, analizar e interpretar las evidencias relacionadas con una pregunta de investigación específica (Kitchenham y Charters, 2007). En nuestro caso se trata de definir, o redefinir, cómo se conceptualiza la educación musical inclusiva (EMI). Para ello se han seguido los pasos establecidos por Guirao, Olmedo y Ferrer (2008).

\subsection{Realización de la búsqueda bibliográfica}

Se realizaron búsquedas automatizadas en las bases de datos de relevancia acreditada en el campo de las ciencias sociales en general y el educativo en particular: ELSEVIER, ERIC, Taylor \& Francis, ProQuest Education Journals, ISOC, PsycARTICLES, SciencieDirect, APA PsycNET. Para acotar la búsqueda y obtener un número razonable de resultados, restringimos la búsqueda mediante filtros y operadores booleanos (Y/AND, O/OR y NO/NOT).

Previo a la búsqueda, la consulta de fuentes impresas permitieron seleccionar las palabras clave que se concretan en la Tabla 1ordenadas por orden alfabético.

Tabla 1. Palabras clave de búsqueda en las bases de datos

\begin{tabular}{|c|c|}
\hline Accesibilidad en educación musical & Accessibility in music education \\
\hline Accesibilidad musical & Musical accessibility \\
\hline Adaptabilidad en educación musical & Adaptability in music education \\
\hline Atención a la diversidad y educación musical & Attention to diversity and music education \\
\hline Características inclusivas de la música & Inclusive features of music \\
\hline Didáctica musical inclusiva & Inclusive musical didactics \\
\hline Educación musical especial & Special music education \\
\hline Educación musical inclusiva & Inclusive music education \\
\hline Enseñanza colaborativa y música & Collaborative teaching and music \\
\hline Estrategias de adaptación en educación musical & Music education adaptation strategies \\
\hline Instrumentos musicales adaptados & Adapted musical instruments \\
\hline Instrumentos musicales adaptados, educación inclusiva & Adapted musical instruments, inclusive education \\
\hline Música e inclusión & Music and inclusion \\
\hline Música y aprendizaje colaborativo & Music and collaborative learning \\
\hline Música y cultura colaborativa & Music and collaborative culture \\
\hline Música y diversidad & Music and diversity \\
\hline Música y resolución de conflictos & Music and conflict resolution \\
\hline Música y sentido de pertenencia & Music and sense of belonging \\
\hline Música y valoración de la diversidad & Music and appreciation of diversity \\
\hline Psicología y educación musical & Psychology and music education \\
\hline Psicología y educación musical especial & Psychology and special music education \\
\hline Psicología y música & Psychology and music \\
\hline Psicopedagogía y música & Psychopedagogy and music \\
\hline Valoración de la diversidad a través de la música & Appreciation of diversity through music \\
\hline
\end{tabular}

\subsection{Selección de la bibliografía de interés: criterios de inclusión y exclusión.}

Los artículos incluidos en esta revisión se han seleccionado teniendo en cuenta los siguientes criterios de inclusión: a) búsqueda limitada al período comprendido entre 2000 y 2019 y b) artículos empíricos y de revisión publicados en español o en inglés. Del total de artículos localizados se eliminaron aquellos que no eran estudios empíricos o de revisión teórica y aquellos cuyo tema, a pesar de estar relacionado con lo investigado, no se ajustaba al ámbito de la educación musical. En esta fase fueron retenidos 48 artículos. 


\subsection{Organización de la información}

Siguiendo la metodología para la síntesis de información sugerida por Cué, Díaz, Díaz y Valdés (2008) se realizó un análisis de los documentos seleccionados mediante tres evaluaciones. En la primera se clasificaron los documentos a partir de una selección de descriptores o palabras clave identificadas como más relevantes, los que aparecen en la Tabla 2, que permitieron organizar los artículos seleccionados. En la segunda evaluación se extrajo el contenido mediante un análisis de contenido deductivo en función de dos categorías de información (adaptabilidad y recurso inclusivo) lo que se refleja en la Tabla 2. La adaptabilidad se define por las posibilidades de accesibilidad universal a los contenidos musicales que se pretenden enseñar, mediante ajustes en la didáctica, en los instrumentos, o en los códigos, pensando en las necesidades técnicas que pueden requerir algunas personas por sus características. La música como recurso inclusivo se define por ofrecer amplias posibilidades de participación y aprendizaje a todo el alumnado, incidiendo en su bienestar personal y social.

Tabla 2. Clasificación de artículos por descriptores y categorías

\begin{tabular}{|c|c|c|c|}
\hline Descriptores & Artículos & Adaptabilidad & Recurso Inclusivo \\
\hline \multirow{13}{*}{ Educación Musical Inclusiva } & $\begin{array}{l}\text { Burnard, Dillon, Rusinek, y Sæther, } \\
(2008)\end{array}$ & & $\checkmark$ \\
\hline & Darrow (2009) & $\checkmark$ & $\checkmark$ \\
\hline & Sabbatella (2008) & $\checkmark$ & $\checkmark$ \\
\hline & Fernández-Carrión Quero (2011) & & $\checkmark$ \\
\hline & Fernández Rodicio (2011) & & $\checkmark$ \\
\hline & Aparicio y León (2018) & & $\checkmark$ \\
\hline & Gutierrez (2016) & & $\checkmark$ \\
\hline & Pérez-Aldeguer (2013) & & $\checkmark$ \\
\hline & Verhagen, Panigada y Morales (2016) & & $\checkmark$ \\
\hline & Vernia y Cantos (2018) & & $\checkmark$ \\
\hline & Palmer (2018) & & $\checkmark$ \\
\hline & Sanahuja, Moliner y Moliner (2019) & & $\checkmark$ \\
\hline & Wong y Chik (2015) & & $\checkmark$ \\
\hline \multirow{18}{*}{ Educación Musical Especial } & Challis (2009) & $\checkmark$ & \\
\hline & Brown et al. (2010) & & $\checkmark$ \\
\hline & Vitoria (2005) & $\checkmark$ & \\
\hline & Eren (2014) & & $\checkmark$ \\
\hline & Herrera et al. (2014) & & $\checkmark$ \\
\hline & Kocabas y Ozeke (2011) & & $\checkmark$ \\
\hline & Lubet (2009) & & $\checkmark$ \\
\hline & OHMI & $\checkmark$ & \\
\hline & Bunt (2003) & & $\checkmark$ \\
\hline & Pellitteri (2000) & & $\checkmark$ \\
\hline & Petruta-Maria (2015) & & $\checkmark$ \\
\hline & Sabbatella (2005) & & $\checkmark$ \\
\hline & Schwartzberg y Silverman (2016) & & $\checkmark$ \\
\hline & Scott (2016) & $\checkmark$ & $\checkmark$ \\
\hline & Skeja (2014) & & $\checkmark$ \\
\hline & $\begin{array}{|llll|}\begin{array}{l}\text { Talavera Jara } \\
(2016)\end{array} & \text { y Gértrudix } \text { Barrio } \\
\end{array}$ & & $\checkmark$ \\
\hline & Rickson y McFerran (2007) & & $\checkmark$ \\
\hline & Wong y Chik (2016) & & $\checkmark$ \\
\hline \multirow{2}{*}{ Música e Inclusión } & Ferreira (2008) & & $\checkmark$ \\
\hline & Gous-Kemp (2013) & & $\checkmark$ \\
\hline
\end{tabular}




\begin{tabular}{|l|l|c|c|}
\hline \multirow{5}{*}{ Psicología y Música } & Blood y Zatorre, (2001) & & $\checkmark$ \\
\hline & Zatorre y Salimpoor (2013) & & $\checkmark$ \\
\hline & Koelsch y Siebel (2005) & & $\checkmark$ \\
\hline & Pantev y Herholz (2011) & & $\checkmark$ \\
\hline \multirow{5}{*}{ Psicopedagogía y Música } & Flix (2000) & & $\checkmark$ \\
\hline & Ho, Cheung, Chan (2003) & & $\checkmark$ \\
\hline & Korsakova-Kreyn y Dowling (2014) & & $\checkmark$ \\
\hline & North y Hargreaves (2008) & & \\
\hline \multirow{3}{*}{$\begin{array}{l}\text { Estrategias de Adaptación encán Musical } \\
\text { Educion }\end{array}$} & Rodríguez de Gil (2000) & $\checkmark$ & $\checkmark$ \\
\hline & Merck y Johnson (2017) & $\checkmark$ \\
\hline & University of Colorado (s.f.) & $\checkmark$ & $\checkmark$ \\
\hline \multirow{5}{*}{ Música y diversidad } & Bond (2017) & $\checkmark$ \\
\hline & Fernández y Epelde (2014) & & $\checkmark$ \\
\hline & Bermell, Bernabé y Alonso (2014) & & $\checkmark$ \\
\hline & Bernabé-Villodre (2012) & $\checkmark$ \\
\hline & Cabedo-Mas (2014a) (2014b) & & $\checkmark$ \\
\hline & Monmany (2004) & & $\checkmark$ \\
\hline
\end{tabular}

Por último, en la tercera evaluación, los extractos del análisis de contenido fueron categorizados de manera inductiva y las subcategorías puestas en relación mediante un mapa conceptual elaborado con el software CmapTools v6.01.01 para proceder a la redacción de los resultados.

\section{Resultados}

En general, parece reforzarse la idea integradora e interdisciplinar que apuntaba Sabbatella (2005) al estimar en la EMI la coexistencia de dos enfoques específicos: el terapéutico y el didáctico. La Figura 1 ilustra esta relación, a modo de síntesis de los resultados obtenidos.

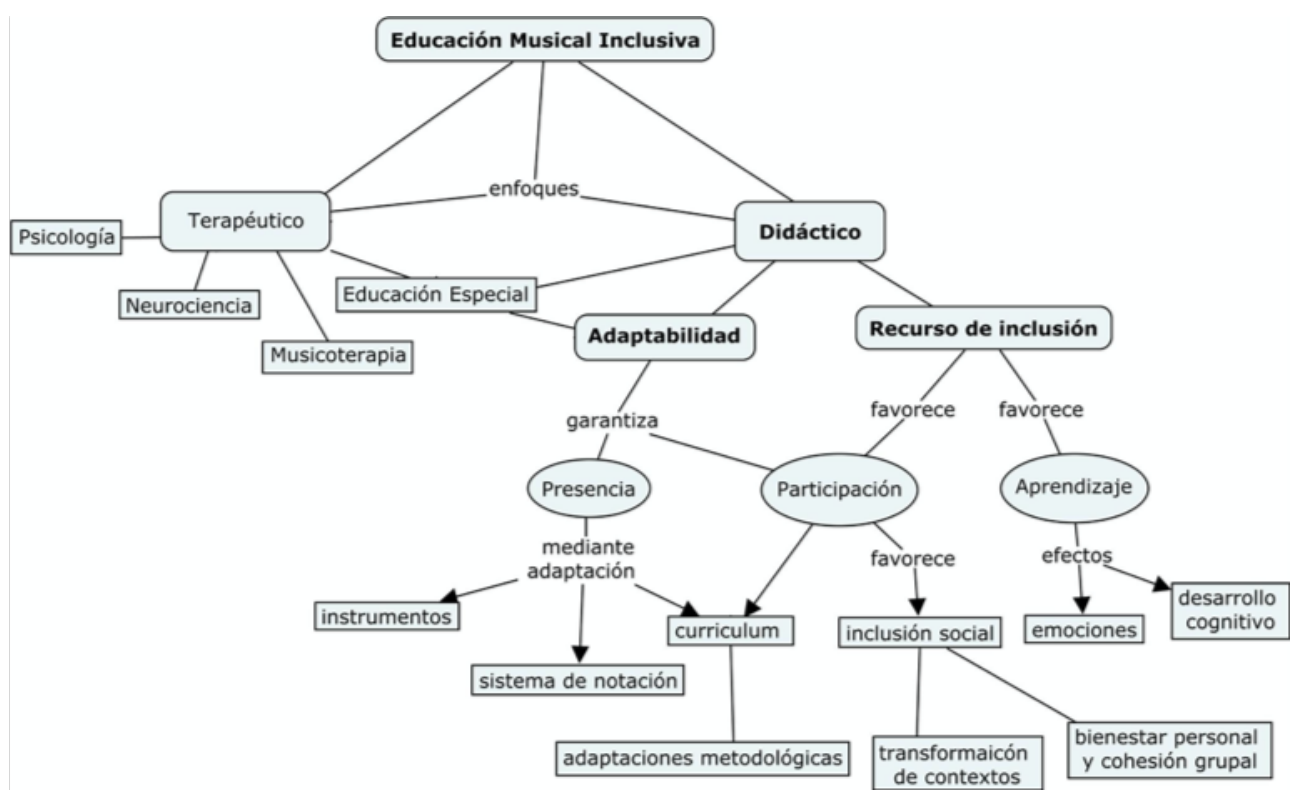

Figura 1. Mapa conceptual para la redefinición de la Educación Musical Inclusiva

\subsection{La EMI como integración de los enfoques terapéutico y didáctico}

Trabajos como el de Skeja (2014) aportan la evidencia de los beneficios de la integración de ambos enfoques mediante la aplicación de un programa terapéutico-didáctico que logra avances en los procesos de planificación y atención, entre otros. 
También, siguiendo estas afirmaciones, de la revisión de la literatura se desprende que la EMI, desde el enfoque más terapéutico, se nutre de las aportaciones de la psicología y la neurociencia, apreciación contemplada también por Sabbatella (2005) y respaldada por estudios como los de Blood y Zatorre (2001), Koelsch y Siebel (2005) y también los de Zatorre y Salimpoor (2013) y Pantev y Herholz (2011).

\subsubsection{Aportaciones de la Musicoterapia y de la Educación Musical Especial}

Investigaciones en el ámbito de la educación musical especial y la psicopedagogía como la de Vitoria (2005) y la de Ho, Cheung y Chan (2003) afirman que la música mejora en los niños las capacidades psicolingüísticas y afecta sistemáticamente al procesamiento de la memoria. También, en esta línea, existen trabajos como el de Schwartzberg y Silverman (2016) que demuestra que la utilización de la música en directo basada en relatos cortos, logró que un grupo de menores con trastorno del espectro autista mejorara las capacidades cognitivas y la comprensión de los relatos. Koelsch y Siebel (2005) profundizan en el campo neurológico proporcionando un marco para el aspecto perceptivo del procesamiento musical. Zatorre y Salimpoor (2013), en un trabajo de revisión, presentan los hallazgos neurocientíficos que incumben a la transición que se produce entre la percepción musical de los patrones de sonido y la respuesta emocional y placentera que de ellos se obtiene. Otros estudios también ahondan en la neurociencia y orientan el ejercicio de la música como una práctica de neuro-rehabilitación, como ocurre en la investigación de Pantev y Herholz (2011). Desde la vertiente más terapéutica de la música y en el ámbito de la educación musical especial, se recogen las aportaciones de la musicoterapia en el trabajo de Rickson y McFerran (2007). Pellitteri (2000) presenta concretamente los beneficios de la musicoterapia contemplando el impacto del arte musical en la cognición. Es también el caso del estudio de Petruta-Maria (2015) en el que se sistematizan métodos eficaces de musicoterapia con la intención de que el alumnado con discapacidad mejore el rendimiento académico. Igualmente la mejora del rendimiento académico es el objetivo por el cual Flix (2000) presenta la musicoterapia como recurso educativo. Estos resultados han de tenerse en cuenta para que no sean desaprovechadas las aportaciones del enfoque más terapéutico.

Desde el enfoque didáctico, como se explica a continuación, también se tienen en cuenta las aportaciones de la Educación Especial por lo que respecta a su carácter compensador de dificultades severas que, si no fueran compensadas, generarían situaciones de desigualdad e injusticia entre el alumnado.

\subsection{EI enfoque didáctico de la EMI.}

\subsubsection{La adaptabilidad en la didáctica musical}

Con respecto a los procedimientos de adaptabilidad en la educación musical, la revisión de la literatura ha mostrado una ausencia significativa de trabajos científicos que traten el tema de forma específica. Ello es probable que sea producto del carácter fundamentalmente práctico y artesanal de la adaptabilidad en el campo de la didáctica de la música. Estas características parecen rehuir de teorizaciones y planteamientos de índole más psicológica o filosófica. Sin embargo se estima conveniente citar la existencia, fuera del ámbito estrictamente científico, de organizaciones benéficas como la One-Handed Musical Instrument Trust (OHMI), institución británica que aboga por la adaptación de los diferentes instrumentos musicales para la inclusión en la interpretación musical de personas con discapacidad física. También es reseñable el trabajo del Center for Inclusive Design and Engineering (CIDE) en Denver, concretamente el documento publicado por la Assistive Technology Partners del College of Engineering and Applied Science que lleva por título Adapting Musical Activities for Persons with Disabilities (University of Colorado, s.f.) que puede considerase como una de las mejores guías prácticas para lograr la adaptabilidad en las actividades musicales y evitar posibles exclusiones. Así, la adaptabilidad aparece en diferentes estudios como un medio para lograr la aplicación de la didáctica especial o de la musicoterapia educativa (Rodríguez de Gil, 2000). Es pues, un modo de garantizar la accesibilidad a la práctica del arte sonoro, y consecuentemente garantizar la presencia en los contextos ordinarios de personas que tradicionalmente han sido privadas de tal experiencia. El trabajo de Merck y Johnson (2017) proporciona un interesante compendio de recursos para realizar adaptaciones de instrumentos y de situaciones de enseñanza-aprendizaje para la educación musical de niños con discapacidad.

Es destacable que, en la EMI, para lograr esa adaptabilidad se recomienda el asesoramiento de un especialista en musicoterapia (Sabbatella, 2005) y se concretan los ámbitos de incidencia en: la elección por parte del docente de actividades musicales que se ciñan a las necesidades del alumnado, los instrumentos musicales que se han de adaptar a las necesidades físicas, o las técnicas específicas que promuevan la interacción y el clima social positivo en el aula. Al parecer, la perspectiva inclusiva de esta autora vería la adaptabilidad no solo como un medio procedimental destinado a población con discapacidad sino que se extendería también a todo tipo de alumnado, rigiéndose por la idea de que tanto los medios instrumentales, como los metodológicos y los relativos a los códigos, se han de adaptar a la realidad individual de cada alumno. Trabajos como el de Challis (2009) confirman esta visión más instrumental.

En el sistema de notación musical la adaptación se materializa utilizando nuevos sistemas musicales, como el utilizado en el trabajo de Vitoria (2005), o incluso adaptando los convencionales a las capacidades del alumnado que se pretende incluir y que presenta dificultades insalvables. En el terreno de la instrumentación musical, la adaptabili- 
dad incidiría en la utilización de instrumentos musicales especiales (Rodríguez de Gil, 2000) o en la adaptación del sistema de ejecución de los instrumentos convencionales.

En el contexto curricular las adaptaciones conciernen tanto al curriculum como a la metodología de la didáctica musical, como ocurre en el trabajo de Scott (2016) que aborda las adaptaciones realizadas por maestros de música para la inclusión de alumnado con TEA en clases de música de primaria. En este contexto cabe destacar la incorporación del aprendizaje cooperativo-colaborativo, pues, según la revisión, es la metodología más adecuada para llevar a cabo una eficaz EMI (Sabbatella, 2008).

\subsubsection{La música como recurso inclusivo}

Por otra parte, la música como recurso inclusivo se vislumbra en trabajos como el de Gous-Kemp (2013). Según este autor, el arte sonoro realiza el papel de artefacto de aprendizaje, teniendo en cuenta la teoría de la mediación semiótica de Vigotsky. Por su parte, trabajos como el de Lubet (2009) aportan a la EMI una perspectiva multicultural musical, que se enfrenta a la concepción musical pedagógica tradicional basada únicamente en la enseñanza de los cánones de la música clásica occidental. Se considera que la pedagogía musical basada solamente en la visión clásica de la música actúa como una barrera de la inclusión del alumnado.

Como recurso inclusivo, la revisión de la literatura apunta que la música favorece la participación y el aprendizaje de todo el alumnado y especialmente de aquellos tradicionalmente excluidos, como el alumnado perteneciente a grupos culturales minorizados o a los denominados colectivos vulnerables.

Algunos de los estudios revisados consideran que la práctica musical favorece la participación y la inclusión social. El recurso del arte musical se utiliza como medio para la total integración de colectivos vulnerables y marginados. Recogen la experiencia sinfónica llevada a cabo por el director de orquesta y pedagogo José Antonio Abréu que creó, con el patrocinio del estado venezolano, El Sistema Nacional de Orquestas y Coros Juveniles e Infantiles de Venezuela (1975). El proyecto de Abréu es objeto de estudio en los trabajos de Verhagen, Panigada y Morales (2016) y de Aparicio y León (2018). En este último se documentan interesantes proyectos en varios países de América Latina como Olodum, Timbalada o Pracatum, el proyecto In Harmony en Reino Unido y en España el proyecto Increscendo o La Ciudad de los Colores, todos ellos favorecedores de la inclusión social. Sin embargo Bond (2017) ofrece una revisión de la literatura y una visión crítica de algunas experiencias centradas en el patrimonio cultural de los estudiantes incorporando materiales multiculturales, muchos de ellos abordando aspectos de raza o etnia, pero pocos que exploren otras manifestaciones culturales como la situación socioeconómica y la religión.

Así pues, el carácter social de una educación musical abierta a todo tipo de alumnado, se pone de manifiesto en gran parte de los trabajos revisados. El trabajo de Brown, Benedett, y Armistead (2010), desarrollado con población infantil en riesgo de exclusión, afirma que ofrece oportunidades educativas importantes para estudiantes de diversos orígenes y necesidades diversas. Para Vernia y Cantos (2018) la música es una estrategia de inclusión en personas adultas que posibilita aprender la lengua, costumbres y tradiciones, sin necesidad de aceptarlas como propias pero reconociéndolas como parte de las sociedad en las que se integran. Aparicio y León (2018) documentan los efectos de la educación musical en la reducción del absentismo escolar, la superación de prejuicios y estereotipos y la mejora de la convivencia e inclusión escolar. También Ferreira (2008) considera que la música, por su carácter lúdico, es un agente poderoso de inclusión y, por su esencia interdisciplinar, un modo de acercarse al conocimiento de diversas realidades.

La literatura también recoge el papel decisivo de la EMI en las transformaciones contextuales y sociales. El trabajo de Fernández-Carrión Quero (2011) aborda la presencia de la música en las comunidades de aprendizaje, modelo inclusivo de participación que adoptan muchos centros educativos. También Gutiérrez (2016) sugiere combinar las ventajas que aportan las citadas comunidades con la música como herramienta de bienestar personal y grupal y por ende, inclusiva.

Así pues, el impacto social de la música en contextos de diversidad está presente también en numerosos trabajos (Bermell, Bernabé y Alonso, 2014; Burnard y Rusinek, 2008; Cabedo- Mas, 2014a, 2014b; Fernández, 2011; Fernández y Epelde, 2014; Pérez-Aldeguer, 2013). De ello podemos deducir que el aspecto esencialmente social de la educación inclusiva se pone de manifiesto en la práctica de la educación musical y es un factor definitorio de la EMI.

Sin embargo, son pocos los trabajos que abordan la práctica de la educación musical inclusiva desde un enfoque crítico basado en la justicia social. Palmer (2018) adopta este enfoque y reivindica la necesidad de abordar temas ausentes en el ámbito de la educación musical como el privilegio y la opresión. Desde la EMI es indispensable plantearse cómo determinados programas perpetúan las brechas y desigualdades debido a factores socioeconómicos y culturales.

Por otra parte, la EMI desde el enfoque didáctico, considera la música como un recurso de inclusión porque favorece el aprendizaje de todo el alumnado. Esto se explica desde la psicología y la psicopedagogía por la clara influencia de la música en las emociones, pues aspectos musicales como la modulación y la tonalidad provocan respuestas de tipo emocional como muestran los estudios de Korsakova-Kreyn y Dowling (2014) y de Fernández Rodicio (2011). Los proyectos inclusivos basados en la música, como el proyecto LOVA de Mary Ruth McGinn, inciden claramente en el aspecto emocional del alumnado (Fernández-Carrión Quero, 2011) al tiempo que permiten articular 
de manera interdisciplinar diferentes áreas curriculares y promueven un aprendizaje funcional y significativo. También permiten, de acuerdo con Sanahuja, Moliner y Moliner (2019), una gestión del aula inclusiva ofreciendo una enseñanza acorde a las necesidades, intereses y ritmos de aprendizaje, generando un clima saludable y un sentido de pertenencia.

Por otra parte, estudios como el de Herrera, Hernández-Candelas, Lorenzo, y Ropp (2014) reportan resultados del aprendizaje musical en el desarrollo general de los niños, incluido el ámbito del lenguaje. Es común, en los estudios revisados, el hecho de relacionar la actividad musical con el desarrollo cognitivo (Sabbatella, 2005; Blood y Zatorre, 2001, Eren (2014) ; Kocabas y Ozeke (2011). El trabajo de Skeja (2014) recoge los resultados positivos de un programa en alumnado de educación infantil con dificultades de aprendizaje. Talavera Jara y Gértrudix Barrio (2016) estudian las mejoras en la comunicación del alumnado con TEA en el marco de las aulas abiertas de centros ordinarios.

\section{Conclusiones}

Tras el análisis de los resultados se pone en evidencia que la Educación Musical Inclusiva se nutre de la confluencia de los enfoques terapéutico y didáctico de la música, siendo el enfoque didáctico el que permite garantizar la presencia, la participación y el aprendizaje de todo el alumnado en contextos educativos ordinarios a través de dos elementos fundamentales: la adaptabilidad y la música como recurso de inclusión.

De los resultados se desprenden las siguientes conclusiones:

1. La adaptabilidad correspondería, tanto a las adaptaciones curriculares como a las de la metodología didáctica y a los recursos necesarios para implementar este tipo de educación, concretando su incidencia en el sistema de notación musical, en la instrumentación y en el currículo. Implica rechazar concepciones de la música que solo valoren positivamente un solo estilo o un solo género musical, o que exclusivamente pretendan objetivos puramente estéticos (Lubet, 2009). Se estima que gracias a las características adaptativas se producirá un acceso universal a los contenidos musicales garantizando no sólo la presencia y acceso, sino también la participación del alumnado, lo cual incide en el bienestar personal y social. La participación del alumnado se consigue mediante la implementación de estrategias didácticas de tipo cooperativo y participativo que logran que se establezcan relaciones más positivas entre el alumnado, incrementando el espíritu de equipo, las relaciones solidarias y comprometidas, el respaldo personal y escolar, la valoración de la diversidad y la cohesión grupal. Estas características favorecen sobremanera la inclusión teniendo en cuenta una adecuada estructuración de los grupos de trabajo, pues su eficacia parece depender de esta característica estructural (Gillies, 2004).

2. La utilización de la música como recurso de inclusión y su repercusión en el aprendizaje está fuertemente influenciado por el enfoque terapéutico musical. Se deduce que el componente emocional existente en el arte sonoro es una de las claves a tener en cuenta por ser la gestión de las emociones uno de los factores que inciden en la inclusión (Fernández Rodicio, 2011) y su tratamiento a través de la música se estima como potenciador y facilitador de óptimos resultados. La revisión parece ofrecer evidencias sobre la afectación positiva de la música en el desarrollo psicolingüístico del alumnado. De ello se deduce que los programas sobre contenidos lingüísticos, reforzados por la utilización de componentes musicales podrían beneficiar significativamente al alumnado, tanto a aquel con problemas en el lenguaje comunicativo y expresivo como a aquel que no los presenta. Sin embargo este es un campo en el que seguir investigando desde el ámbito de la didáctica de la música.

3. Las características de la EMI, conducen inevitablemente al planteamiento de las particularidades del principal artífice o dinamizador de la misma en el contexto del aula, en este caso la figura del docente musical e inclusivo. Aunque no se encuentran investigaciones específicas sobre el tema, sí que se aborda en algunos estudios que apuntan características sobre su perfil y estilo. De acuerdo con Sabbatella (2008) el educador musical inclusivo requiere estar dotado de un papel activo y participativo en la faceta musical, capacitado para dar un trato equitativo a todo el alumnado, ágil y creativo para la gestión de problemas, conocedor de las características del alumnado y capaz de elegir, diseñar y adaptar las actividades. A estas características cabría añadir, atendiendo a la presente revisión, ciertos conocimientos psicopedagógicos necesarios para orientar la acción inclusiva desde el aspecto musical. Si bien es cierto que la música facilita la implementación de acciones inclusivas, su correcto aprovechamiento depende de una hábil y consciente gestión en la que el conocimiento musical y el psicopedagógico del docente van ligados. Una simple pero ilustrativa prueba de ello es la interpretación en ensamble de piezas musicales. Per se, posee características inclusivas ya que todo el alumnado ha de intervenir en la interpretación, pero, no obstante, será determinante la asignación de las partes instrumentales teniendo en cuenta las habilidades de los alumnos e incluso, con este criterio, contemplar sus posibles adaptaciones.

Así pues, podemos concluir que la EMI es un ámbito emergente de la educación musical que atiende principalmente a dos aspectos fundamentales: facilitar la accesibilidad para el aprendizaje de los contenidos musicales y ser, en sí misma, un recuso para la inclusión. Teniendo en cuenta que la educación inclusiva se ha ido gestando como un enfoque alternativo al médico-terapéutico de la educación especial, consideramos que algunas de las aportaciones de esta última pueden ser integradas a la práctica de la EMI bajo el marco filosófico y pedagógico de la escuela inclusi- 
va, es decir, prestando especial atención a los procedimientos de la didáctica de la música que garanticen la presencia, la participación y el aprendizaje de todo el alumnado en el aula ordinaria.

Los resultados de esta revisión evidencian una compleja convergencia de aspectos disciplinares en la EMI. A juicio de la presente revisión se abren nuevas líneas a explorar en futuras investigaciones como son: el abordaje de la EMI desde un enfoque crítico y de justicia social, los resultados en los aprendizajes del alumnado en contextos inclusivos y la formación del docente musical inclusivo. La colaboración de todos los agentes educativos, junto con las oportunas orientaciones de los especialistas en los campos involucrados, es el mayor garante de que la presumible interdisciplinariedad de la EMI no se relegue a una elucubración teórica sino que se transforme en una realidad con características prácticas, que repercutan positivamente en el alumnado.

\section{Referencias bibliográficas}

Aparicio, J. M., y León, M. M. (2018). La música como modelo de inclusión social en espacios educativos con alumnado gitano e inmigrante. Revista Complutense de Educación, 29(4), 1091-1108.

Bermell, M. Á., Bernabé, M., y Alonso, V. (2014). Diversidad, música y competencia social y ciudadana: Contribuciones de la experiencia musical. Arbor, 190(769), a164.

Bernabé-Villodre, M. (2012). La comunicación intercultural a través de la música. Espiral, 29(1), 69-73.

Blood, A. J., y Zatorre, R. J. (2001). Intensely pleasurable responses to music correlate with activity in brain regions implicated in reward and emotion. Proceedings of the National Academy of Sciences, 98(20), 11818-11823.

Bond, V.L. (2017). Culturally responsive education in music education: a literature review. Contributions to Music Education, 42, 153-180.

Brown, E. D., Benedett, B., y Armistead, M. E. (2010). Arts enrichment and school readiness for children at risk. Early Childhood Research Quarterly, 25(1), 112-124.

Bunt, L. (2003). Music therapy with children: A complementary service to music education? British Journal of Music Education, 20(2), 179-195.

Burnard, P., Dillon, S., Rusinek, G., \& Saether, E. (2008) Inclusive pedagogies in music education: a comparative study of music teachers' perspectives from four countries. International Journal of Music Education, 26(2), 109-126.

Cabedo-Mas, A. (2014a). La música y su aprendizaje en la construcción de culturas para hacer las paces. Cultura de Paz, 64 (20), $10-16$.

Cabedo-Mas, A. (2014b). La música como proyecto socioeducativo. En A. Giráldez, Didáctica de la música en primaria. (pp. 101- 122), Madrid: Síntesis

Calleja-Bautista, M.; Sanz-Cervera, P. y Tárraga-Mínguez, R. (2016) Efectividad de la musicoterapia en el trastorno de espectro autista: estudio de revisión. Papeles del Psicólogo, 37(2), 152-160

Challis, B. (2009). Technology, accessibility and creativity in popular music education. Popular Music, 28(3), 425-431

Cué, M., Díaz, G., Díaz, A. G., \& Valdés, M. C. (2008). El artículo de revisión. Revista Cubana de Salud Pública, 34(4), 1-9.

Darrow, A. (2009). Barriers to Effective Inclusion and Strategies to Overcome Them. General Music Today, 22(3), 29-31.

Del Barrio, Sabbatella y Mercadal (2019). Musicoterapia en Educación: un proyecto de innovación orientado a la inclusión del alumnado con necesidades educativas especiales. Revista Música Hodie, 19, 1-13

Eren, B. (2014). Use of Music in Special Education and Application Examples from Turkey. Procedia - Social and Behavioral Sciences, 116, 2593-2597.

Fernández-Carrión Quero, M. (2011). Proyectos musicales inclusivos. Tendencias Pedagógicas, 17, 74-82.

Fernández Rodicio, C. I. (2011). La inteligencia emocional como estrategia educativa inclusiva. Innovación Educativa, 21, 133150.

Fernández, S. S., y Epelde, A. (2014). Cultura de paz y educación musical en contextos de diversidad cultural. Revista de Paz y Conflictos, 7(7), 79-97.

Ferreira, C. (2008). Música, interdisciplinariedad e inclusión social. El friso sonoro como propuesta de aplicación en el aula. Música, 127-136.

Flix, C. T. (2000). El recurso educativo de la musicoterapia. Revista Pedagógica Maestros de Lima. 5, 1-12.

Gfeller, K. (2000). Musicoterapia en el Entorno Educativo. En Davis, William; Gfeller, Kate y Thauth, Michael: Introducción a la Musicoterapia: Teoría y Práctica, Barcelona: Boileau.

Gillies, R. M. (2004). The effects of cooperative learning on junior high school students during small group learning. Learning and Instruction, 14(2), 197-213.

Gous-Kemp, C. (2013). The creative use of music in inclusive education: Bringing harmony to the classroom. Education as Change, 18(1), 191-206.

Guirao, J. A., Olmedo, A., y Ferrer, E. (2008). El artículo de revisión. Revista Iberoamericana de Enfermeria Comunitaria, 1(1)125.

Gurgenidze, M,. \& Mamuladze, N. (2017) The Role of Cultural Events in Rehabilitation, Therapy and Education of People with Special Needs. Journal of Education and Practice, 13(8) 35-38.

Gutiérrez, A. M. (2016). La música en el ámbito educativo: las comunidades de aprendizaje. International Journal for 21 St. Century Education, 3(1), 15-24. 
Herrera, L., Hernández-Candelas, M., Lorenzo, O., y Ropp, C. (2014). Influencia del entrenamiento musical en el desarrollo cognitivo y lingüístico de niños de 3 a 4 años. Revista de Psicodidactica, 19(2), 367-386.

Ho, Y.C., Cheung, M. C., Chan, A. S. (2003). Music training improves verbal but not visual memory: Cross-sectional and longitudinal explorations in children. Neuropsychology, 17(3), 439-450.

Howes, A., Davies, S. M. B., \& Fox, S. (2009). Improving the Context for Inclusion: Personalising teacher development through collaborative action research. (1 ed.) Routledge.

Kitchenham, B. A., \& Charters, S. (2007). Guidelines for performing Systematic Literature Reviews in Software Engineering. Version 2.3 (EBSE-2007-01). Retrieved from http://www.elsevier.com/_data/promis_misc/525444systematicreviewsguide. pdf

Kocabas, E. O., \& Ozeke, S. (2011). Using Music and Musical Activities in Special Education: Developments in Turkey. Online Submission, 1(1), 73-79.

Koelsch, S. y Siebel, W. A. (2005). Towards a neural basis of music perception. Trends in Cognitive Sciences, 9(12), 578-584.

Korsakova-Kreyn, M., \& Dowling, W. J. (2014). Emotional processing in music: Study in affective responses to tonal modulation in controlled harmonic progressions and real music. Psychomusicology: Music, Mind, and Brain, 24(1), 4-20.

Leggieri M., Thaut, M.H., Fornazzari, L., Schweizer T.A., Barfett, J. Munoz, D.G. \& Fischer, C.E. (2019) Music Intervention Approaches for Alzheimer's Disease: A Review of the Literature. Front. Neurosci. 13,132. doi: 10.3389/fnins.2019.00132

Llopis, B.E. (2011). Hacer música...para comprometerse con la realidad. Eufonía, 51, 34-43.

Lubet, A. (2009). The inclusion of music/the music of inclusion. International Journal of Inclusive Education, 13(7), 727-739.

McFerran, K. S., \& Rickson, D. (2014). Community music therapy in schools: Realigning with the needs of contemporary students, staff and systems. International Journal of Community Music, 7(1), 75-92.

Merck, K., \& Johnson, R.M. (2017). Music Education for Students with Disabilities: A Guide for Teachers, Parents, and Students. The Corinthian:18 (6), 1-16. Recuperado de: https://kb.gcsu.edu/thecorinthian/vol18/iss1/6

Monmany, M. (2004). Acerca de la educación musical. Revista Electrónica de LEEME. 13(5), 1-23.

North, A. C., \& Hargreaves, D. J. (2008). The social and applied psychology of music. Oxford: Oxford University Press.

Ouellet, S. y Poliquin, N. (2012) La musicothérapie au service du développement de l'enfant ayant un trouble déficitaire de l'attention avec hyperactivité (TDAH). Canadian Journal of Music Therapy, 18(1) 79-90

Palmer, E. S. (2018). Literature review of social justice in music education: Acknowledging oppression and privilege. Update: Applications of Research in Music Education, 36(2), 22-31. doi:10.1177/8755123317711091.

Pantev, C., \& Herholz, S. C. (2011). Plasticity of the human auditory cortex related to musical training. Neuroscience and Biobehavioral Reviews, 35(10), 2140-2154.

Pellitteri, J. (2000). The consultant's corner: "Music Therapy in the Special Education Setting." Journal of Educational and Psychological Consultation, 11(3-4), 379-391.

Pérez-Aldeguer, S. (2010). Inclusión social en el aula a través de la música. En: M. Sanchiz, M. Marti y I. Cremades, ed., Orientación e intervención educativa. Retos para los orientadores del S. XXI (pp.637-647), Valencia: Tirant lo Blanch.

Pérez-Aldeguer, S. (2013). El desarrollo de la competencia intercultural a través de la educación musical: Una revisión de la literatura. Revista Complutense de Educacion, 24(2), 287-301.

Pérez, J.E. y del Basto, L.M. (2011). Formación ciudadana por medio de la música andina colombiana: hacia la consolidación del sentido de pertenencia y la identidad cultural. Revista Perspectivas Educativas, 4, 241 - 261

Pérez, A., y Sabbatella, P. (2014). Musicoterapia en Educación: Proyecto de Trabajo Colaborativo para atender alumnado con Trastorno Generalizado del Desarrollo. Libro de actas del V Congreso Nacional de Musicoterapia. Orquestando la Musicoterapia

Petruta-Maria, C. (2015). The Role of Art and Music Therapy Techniques in the Educational System of Children with Special Problems. Procedia - Social and Behavioral Sciences, 187, 277-282.

Rickson, D., y McFerran, K. S. (2014). Creating music cultures in the schools. Barcelona Publishers.

Rickson, D.J., \& McFerran, K. (2007). Music Therapy in Special Education. Where are we now? Kairaranga, 8(1), 40-37

Rodríguez de Gil, G. (2000). Las adaptaciones de instrumentos musicales como ayuda para la musicoterapia en parálisis cerebral. Asociación Parálisis Cerebral APPC, 17, 117-125.

Sabbatella, P. (2005). Intervención musical en el alumnado con necesidades educativas especiales delimitaciones conceptuales desde la pedagogía musical y la musicoterapia. Tavira, 21, 123-139.

Sabbatella, P. (2008). Educación Musical Inclusiva: Integrando perspectivas desde la Educación Musical y la Musicoterapia Educativa. Música. Arte. Diálogo. Civilización, 256-268.

Sanahuja, A., Moliner, O., y Moliner, L. (2019). Gestión del aula inclusiva a través del proyecto LÓVA: la ópera como vehículo de aprendizaje. Revista Electrónica Complutense De Investigación En Educación Musical - RECIEM, 16, 3-19. https://doi. org/10.5209/reciem.62101

Schwartzberg, E. T., \& Silverman, M. J. (2016). Effects of a Music-Based Short Story on Short- and Long-Term Reading Comprehension of Individuals with Autism Spectrum Disorder: A Cluster Randomized Study. The Arts in Psychotherapy, 48, 54-61.

Scott, S. (2016). The Challenges of Imitation for Children with Autism Spectrum Disorders with Implications for General Music Education. National Association for Music Education, 34(2), 13-20.

Skeja, E. (2014). The Impact of Cognitive Intervention Program and Music Therapy in Learning Disabilities. Procedia - Social and Behavioral Sciences, 159, 605-609. 
Talavera Jara, P., y Gértrudix Barrio, F. (2016). El uso de la musicoterapia para la mejora de la comunicación de niños con Trastorno del Espectro Autista en Aulas Abiertas Especializadas. Revista Complutense de Educación, 27(1), $257-284$.

University of Colorado (Sin Fecha). Adapting Musical Activities for Persons with Disabilities. Recuperado de: http://www. ucdenver.edu/academics/colleges/medicalschool/programs/atp/Documents/AdaptingMusicalActivitiesforPersonswithDisabilities.pdf

Verhagen, F., Panigada, L., y Morales, R. (2016). El Sistema Nacional de Orquestas y Coros Juveniles e Infantiles de Venezuela: un modelo pedagógico de inclusión social a través de la excelencia musical. Revista Internacional de Educación Musical, 4 , 35-46.

Vernia, A. M. y Cantos, F. J. (2018). La inclusión educativa y social desde la música y la palabra. Dedica. Revista de Educação e Humanidades, 14, 11-19. doi: 10.30827/dreh.v0i14.7477

Vitoria, J. R. (2005). Educación Musical y Desarrollo Psicolingüístico de Personas con Necesidades Educativas Especiales. Revista de Psicodidáctica, 10, 17-26.

Wong, M. W. Y., y Chik, M. P. Y. (2015). Learned helplessness in inclusive music classrooms: Voices of Hong Kong primary schools music teachers. International Journal of Inclusive Education, 19(9), 965-977.

Wong, M. W. y Chik, M. P. (2016). Teaching students with special educational needs in inclusive music classrooms: experiences of music teachers in Hong Kong primary schools. Music Education Research, 18(2), 195-207.

Zatorre, R. J., y Salimpoor, V. N. (2013). From perception to pleasure: music and its neural substrates. Proceedings of the National Academy of Sciences of the United States of America, 110 Suppl, 10430-10437. 